Interferon interference obstructs ducts

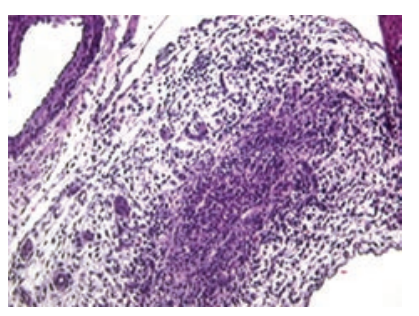

Biliary atresia, a disease in which bile flow is increasingly impeded through progressive obstruction of extrahepatic bile ducts, occurs within the first few weeks of life and is the primary cause of chronic liver disease in children. Liver transplant is ultimately the only treatment, as little is known about the underlying pathogenic mechanisms. The disease state is heterogeneous, but all biliary atresia cases are characterized by inflammatory injury to the bile ducts. Jorge Bezerra and colleagues used a mouse model of a rotavirus-induced biliary atresia to investigate the hypothesis that IFN- $\gamma$ is involved in the development of this disease (pages $322-329$ ). Infecting mice with rotavirus within the first 24 hours of life generates the pathology of biliary atresia. The authors found that in infected mice, in contrast to wild-type mice, IFN- $\gamma$-producing lymphocytes invaded the portal tracts and that IFN- $\gamma$ production was markedly increased in several $\mathrm{T}$ lymphocytes. Infecting mice that lacked IFN- $\gamma$ suppressed T lymphocyte targeting of lymphocytes to the bile ducts, but administering recombinant IFN- $\gamma$ following infection in these IFN $\gamma^{-/-}$mice, resulted in bile duct blockage. These data identify IFN- $\gamma$ as a key player underlying this deadly condition and suggest potential new investigative and therapeutic directions for the study of bile duct obstruction.

\section{Wasting away in muscle-ville}

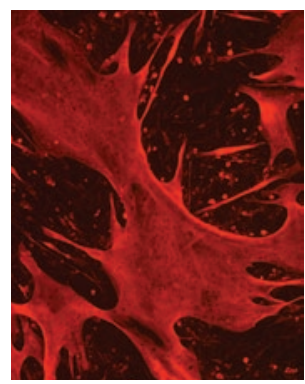

Cachexia is a condition that is characterized by skeletal muscle wasting. Loss of muscle tissue occurs in over half of cancer patients and is the direct cause of nearly one-third of cancer deaths. Cytokines and tumor factors, such as TNF- $\alpha$ and IFN- $\gamma$, are known to be involved in this process, but their targets in the muscle remain largely unknown.

Using in vitro and in vivo models, Denis Guttridge and colleagues investigated the proteins in muscle that are affected in the presence of cytokines (pages 370-378). The authors examined myosin heavy chain, actin, troponin, and tropomyosin as model protein targets of wasting in both cell culture and tumor models of cachexia in mice. They found a striking specificity for the loss of myosin heavy chain only. Intriguingly, TNF- $\alpha / \mathrm{IFN}-\gamma$-dependent loss of myosin heavy chain occurred through an RNAdependent mechanism in cell culture and through a protein degradation process involving the ubiquitin-dependent proteasome pathway in tumor-bearing mice. The authors suggest that the mechanism for myosin heavy chain downregulation may be related to the cachexia factor mediating muscle wasting.

\title{
Leptin regulates prohormone processing
}

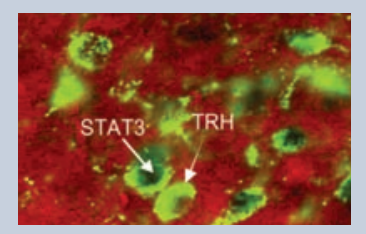

Leptin regulation of energy balance works through the activity of several neuropeptides. Many of these neuropeptides undergo proteolytic processing before they are active. Eduardo Nillni and colleagues theorized that leptin might regulate prohormone-processing enzymes in addition to directly regulating neuropeptide concentration. They tested their hypothesis by examining leptin's effect on prohormone convertase 1 and 2 (PC1 and PC2), enzymes required for thyrotopin-releasing hormone (TRH) maturation (pages 357-369). In primary hypothalamic neuronal cultures, leptin treatment led to a significant increase in PC1 and PC2 RNA and protein levels. Analysis in 293 T cells and in rats showed leptin increased PC1and PC2-promoter activity through a STAT3-dependent mechanism. Rats that were starved and thus had low leptin serum levels likewise had reduced PC1 and PC2 RNA and protein levels that increased with exogenous leptin administration. Consistent with lower levels of PC1 and PC2, concentration of mature TRH was also reduced in fasted rats. Addition of leptin increased mature TRH levels and TRH prohormone PC1 and PC2 cleavage activity. These data provide evidence for an additional key regulatory point in the control of energy balance through leptin and may suggest novel therapeutic strategies for treatment of obesity and thyroid axis-related diseases.

\section{Putting the $\mathbf{B}$ cell in bowel disease}

Inflammatory bowel disease research has focused primarily on $\mathrm{CD} 4^{+} \mathrm{T}$ cell-subset function, and investigation of other immune cells in the pathogenicity of these diseases has just begun. Klaus Ley and colleagues investigate the role B cells may play in the development of ileitis in SAMP1/YitFc mice, a model for human Crohn disease (pages 389-398). Examining the mesenteric lymph nodes (MLNs) in SAMP1/YitFc mice, the authors found an expanded B cell population as well as an increased level of serum B cell-produced IgA compared with what was found in wild-type mice. The severity of ileitis in these mice correlated with MLN B cell number. There was also an increased number of MLN $\alpha_{\mathrm{E}} \beta_{7}$-integrin-expressing $\mathrm{CD}^{+} \mathrm{T}$ cells, and these cells, although they have a regulatory phenotype, were unable to prevent ileitis development when adoptively transferred into SCID mice. Cotransference of B cells increased ileitis severity in these mice. The authors' work here provides evidence for important interactions between $\mathrm{T}$ and B cells as well as in B cell function in the development of a Crohn-like mouse ileitis model. 